\title{
Prognostic value of sleep-through blood pressure surge in patients with essential hypertension
}

\section{Introduction}

Blood pressure shows diurnal variations, with decreases during sleep and surges in the morning. ${ }^{1}$ An excessive increase in morning systolic blood pressure is termed an exaggerated morning surge. Excessive increases can be detected by measuring either the sleepthrough surge or pre-waking surge. The sleep-through surge is defined as the difference between the mean of two systolic blood pressure readings in the $2 \mathrm{~h}$ after waking and the mean of 3 nighttime readings (the lowest reading, the reading just before and the reading just after). ${ }^{2}$ Ambulatory blood pressure monitoring is required to examine the difference between sleep and awake readings. Hypertensive patients with elevated morning blood pressure surges have an increased risk of cardiovascular events and target organ damage..$^{3-8}$ Therefore, measurement of blood pressure in the early morning and soon after arising can provide clinically relevant data on these patients. The aim of this study was to investigate the utility of the sleep-through surge in predicting cardiac events in patients with essential hypertension.

\section{Material and methods}

\section{Study population}

This was a prospective observational study that included 160 adult participants $>18 \mathrm{y}$. recruited from the outpatient clinic of hypertension primary heath care centers in Dammam, Saudi Arabia from January 2013 to May 2013 and were followed up for $5 \mathrm{y}$. Nineteen of the 160 patients were excluded because they did not meet the inclusion criteria. Follow-up information data of 8 patients were missed. Thus, the final study enrolled 133 patients. The inclusion criteria were patients who had primary hypertension with systolic blood pressure $\geq 140 \mathrm{mmHg}$ or diastolic blood pressure $\geq 90 \mathrm{mmHg}$. Office blood pressure was measured in a sitting position after resting for $5 \mathrm{~min}$. For patients newly diagnosed with primary hypertension, the average of two measurements on different days was used. The exclusion criteria were secondary hypertension, a history of coronary artery disease (CAD), previous coronary vascularization, heart failure, and pregnancy. The study was approved by the local ethics committee, and all the subjects provided signed informed consent before participation in the study.

\section{Ambulatory blood pressure monitoring}

A Tonoport V device (GE healthcare, 8200 W Tower Ave, Milwaukee, WI, USA) was used for 24-h blood pressure monitoring. Blood pressure readings were recorded by protocol P3. In accordance with this protocol, blood pressure was recorded every $30 \mathrm{~min}$ in the daytime (from $6 \mathrm{AM}$ to $10 \mathrm{PM}$ ) and every $60 \mathrm{~min}$ at night (from $10 \mathrm{PM}$ to $6 \mathrm{AM}$ ). Maximal inflation pressure during the daytime phase was $250 \mathrm{mmHg}$, and it was $220 \mathrm{mmHg}$ during the nighttime phases. The mean daytime, nighttime, and 24-h systolic and diastolic pressures were recorded. The sleep-through surge was calculated by subtracting the morning systolic blood pressure (mean of readings in the $2 \mathrm{~h}$ after waking) from the lowest nocturnal systolic blood pressure (mean of 3 nighttime readings; the lowest, the reading just before and the reading just after).

\author{
Volume II Issue 4 - 2018 \\ Mohamed Yahia,' Shereen Mahrous ${ }^{2}$ \\ 'Department of Cardiology, faculty of Medicine, University of \\ Menoufia, Egypt \\ ${ }^{2}$ Department of Family Medicine, Primary Health Care Center, \\ Dammam, Ministry of Health, Saudi Arabia
}

Correspondence: Mohamed Yahia, Department of Cardiology, faculty of Medicine University of Menoufia, Egypt, Tel +00201005362162, Email m_yahia2000@yahoo.com

Received: June 23, 2018 | Published: July 18, 2018

\section{Resting two-dimensional echocardiography}

Resting echocardiography was performed for each patient, with measurements of cardiac dimensions, left ventricular ejection fraction (LVEF \%), and the LV mass index. A value of $95 \mathrm{~g} / \mathrm{m}^{2}$ in women and $115 \mathrm{~g} / \mathrm{m}^{2}$ in men was used to identify the upper limits of normal LV mass. ${ }^{9}$

\section{Laboratory blood tests}

A venous blood sample was drawn from each patient after fasting for $10 \mathrm{~h}$ for analysis of serum creatinine, fasting blood sugar, glycosylated hemoglobin (HbA1c), and lipid profiles.

\section{Follow up for cardiac events}

After ambulatory blood pressure monitoring, the patients' medical records were reviewed periodically for the occurrence of cardiac events (acute coronary syndrome, coronary revascularization, and sudden death) for $5 \mathrm{y}$.

\section{Statistical analysis}

Data analysis was done using the IBM SPSS software package, version 20.0 (IBM Corp, Armonk, NY, USA). Normal distribution of the data was investigated using Kolmogorov-Smirnov, ShapiroWilk, and D'agostino tests. Group statistical comparisons were done using a chi-squared test (Fisher's or Monte Carlo) for categorical variables. The Student's $t$-test and Mann-Whitney $U$ test were used to compare the statistical difference between variables that followed a Gaussian distribution. Spearman's and Pearson's coefficient tests were conducted to study correlations between the variables. $P$ values $\leq 0.05$ indicated a statistically significant difference. The diagnostic cutoff value of the sleep-trough surge was determined by a receiver operating characteristic curve (ROC). Logistic regression analysis was conducted to detect factors associated with cardiac events.

\section{Results}

This study included 72 males (54\%) and 61 females (46\%) with 
essential hypertension for a mean duration of $6.5 \pm 3.4 \mathrm{y}$. The mean clinical systolic blood pressure was $151.3 \pm 10.8 \mathrm{mmHg}$, and the mean clinical diastolic blood pressure was $91.6 \pm 7.1 \mathrm{mmHg}$. In the study, $34(25.5 \%)$ subjects were diabetics, $38(28.6 \%)$ were dyslipidemic and $36(27 \%)$ were smokers. Sixteen $(12 \%)$ patients had a family history of CAD.

The patients were classified into two groups according to the development of cardiac events during follow up. Cardiac events occurred in $19(14.3 \%)$ patients (group 1). Of these, $11(8.3 \%)$ developed unstable angina/non-ST segment elevation myocardial infarction, 8 (6\%) developed ST elevation myocardial infarction, and $16(12 \%)$ had coronary revascularization. No cardiac deaths were reported. Patients who developed cardiac events were older than those who did not have adverse events ( $59.6 \pm 7.8$ vs. $54.9 \pm 9$ y). They also had a higher frequency of diabetes mellitus (DM) (47.4\% vs. $21.9 \%$, $p=0.043)$ and a higher LV mass index (112.6 \pm 17.8 vs. $104.1 \pm 15.8 \mathrm{~g} /$ $\mathrm{m}^{2}, \mathrm{p}=0.035$ ). There were no significant differences among the groups with regard to body mass index, smoking, and dyslipidemia. There were also no significant differences among the groups in the duration of hypertension and antihypertensive medications Table $1 \&$ Table 2.

Table I Patient's characteristics

\begin{tabular}{llll}
\hline & $\begin{array}{l}\text { Group I } \\
(\mathbf{n}=19)\end{array}$ & $\begin{array}{l}\text { Group 2 } \\
(\mathbf{n}=114)\end{array}$ & $\mathbf{p}$ \\
\hline Age & $59.6 \pm 7.8$ & $54.9 \pm 8.9$ & 0.034 \\
Range & $44-70$ & $36-77$ & \\
Sex & & & \\
Male & $1 \mathrm{I}(57.9 \%)$ & $6 \mathrm{I}(53.5 \%)$ & 0.722 \\
Female & $8(42.1 \%)$ & $53(46.5 \%)$ & \\
BMI, kg/m & $28.6 \pm 3.2$ & $28.2 \pm 4.4$ & 0.679 \\
Range & $24-35$ & $21-38$ & \\
Duration of HTN (years) & $7.2 \pm 3.6$ & $6.4 \pm 3.5$ & 0.247 \\
Range & $2-15$ & $1-26$ & \\
Diabetes Mellitus (n.,\%) & $9(47.4 \%)$ & $25(21.9 \%)$ & 0.043 \\
Dislipedemia (n.,\%) & $8(42.1 \%)$ & $30(26.3 \%)$ & 0.158 \\
Smoker (n.,\%) & $6(31.6 \%)$ & $30(26.3 \%)$ & 0.633 \\
Family history of CAD & $4(21 \%)$ & $12(10 \%)$ & 0.192 \\
(n.,\%) & & & \\
Antihypertensive drugs & & & \\
Diuretics (n.,\%) & $1 \mathrm{I}(57.9 \%)$ & $45(39.5 \%)$ & 0.132 \\
B-Blockers (n., \%) & $9(47.4 \%)$ & $49(43 \%)$ & 0.721 \\
CCBs (n.,\%) & $6(31.6 \%)$ & $28(24.6 \%)$ & 0.572 \\
ACEls (n.,\%) & $7(36.8 \%)$ & $32(28.1 \%)$ & 0.437 \\
\hline
\end{tabular}

HTN: Hypertension, BMI: Body mass index, CAD: Coronary artery disease.

Table 3 shows the differences in ambulatory blood pressure monitoring data between the two groups. Patients who developed cardiac events had higher $24 \mathrm{~h}$, daytime, nighttime, and morning systolic blood pressures than those who did not experience cardiac events. They also had elevated daytime and nighttime diastolic blood pressures. The sleep-through surge was significantly higher in patients who developed cardiac events as compared with those who did not $(40.2 \pm 14.5$ vs. $18 \pm 9.8, p<0.00)$ (Figure 1$)$.

The sleep-through surge and risk factors associated with CAD (age, DM, LV mass index, and glycosylated $\mathrm{HbA} 1 \mathrm{c} \%$ ) were evaluated in a multivariate logistic regression analysis. An increased sleep-through surge and DM were independent risk factors for the presence of CAD (for sleep-trough surge: odds ratio: 1.27; confidence interval: 1.033 $1.61 ; p<0.05)$, as shown in Table 4.

Table 2 Laboratory and echocardiographic findings of the studied patients

\begin{tabular}{|c|c|c|c|}
\hline & $\begin{array}{l}\text { Group I } \\
(n=19)\end{array}$ & $\begin{array}{l}\text { Group } 2 \\
(n=|| 4)\end{array}$ & $\mathbf{p}$ \\
\hline Total cholesterol, mg/dl & $197.7 \pm 29.7$ & $192.8 \pm 31.3$ & 0.527 \\
\hline LDL-C, mg/dl & $132.4 \pm 28.5$ & $126.4 \pm 30.3$ & 0.427 \\
\hline HDL-C, mg/dl & $37.9 \pm 7.1$ & $39.1 \pm 6.8$ & 0.489 \\
\hline $\mathrm{TG}, \mathrm{mg} / \mathrm{dl}$ & $138 \pm 39.4$ & $137.6 \pm 47.3$ & 0.668 \\
\hline $\mathrm{HbAlc} \%$ & $6.3 \pm 1$ & $5.7 \pm 0.8$ & 0.003 \\
\hline Creatinine, $\mathrm{mg} / \mathrm{dl}$ & $0.9 \pm 0.21$ & $0.89 \pm 0.20$ & 0.363 \\
\hline LV diastolic dimension, $\mathrm{cm}$ & $5.1 \pm 0.4$ & $5 \pm 0.4$ & 0.853 \\
\hline LV systolic dimension, $\mathrm{cm}$ & $3 \pm 0.4$ & $3.1 \pm 0.4$ & 0.365 \\
\hline LV ejection fraction,\% & $61.6 \pm 4$ & $62.6 \pm 5.1$ & 0.442 \\
\hline LV mass index, $g / \mathrm{m}^{2}$ & $112.6 \pm 17.8$ & $104.1 \pm 15.8$ & 0.035 \\
\hline
\end{tabular}

HDL-C: High density lipoprotein cholesterol, LDL-C: Low density lipoprotein cholesterol, TG: Triglycerides, HbA : Glycosylated hemoglobin, LV: Left ventricle.

Table 3 Comparison between the two studied groups according to blood pressure data

\begin{tabular}{llll}
\hline & $\begin{array}{l}\text { Group I } \\
(\mathbf{n}=19)\end{array}$ & $\begin{array}{l}\text { Group 2 } \\
(\mathbf{n}=114)\end{array}$ & $\mathbf{p}$ \\
\hline Clinic systolic BP, mmHg & $156.5 \pm 11.3$ & $150.4 \pm 10.5$ & 0.022 \\
24-Hour systolic BP, mmHg & $144.4 \pm 7.6$ & $137.2 \pm 9.5$ & 0.002 \\
Daytime systolic BP, mmHg & $150.7 \pm 8.6$ & $141.3 \pm 10.5$ & $<0.001$ \\
Night systolic BP, mmHg & $130.1 \pm 12.3$ & $126.5 \pm 9.6$ & 0.151 \\
Lowest sleep systolic BP, mmHg & $112.8 \pm 13.0$ & $114.6 \pm 10.6$ & 0.508 \\
Morning systolic BP, mmHg & $153 \pm 13.9$ & $132.7 \pm 13.1$ & $<0.001$ \\
Nighttime fall in systolic BP, \% & $13.4 \pm 8.6$ & $10.3 \pm 6.1$ & 0.059 \\
Sleep-through surge, mmHg & $40.2 \pm 14.5$ & $18 \pm 9.8$ & $<0.001$ \\
Clinic diastolic BP, mmHg & $94.1 \pm 9.5$ & $91.3 \pm 6.6$ & 0.120 \\
24-Hour diastolic BP, mmHg & $86.9 \pm 5$ & $84.5 \pm 5.5$ & 0.075 \\
Daytime diastolic BP, mmHg & $87.9 \pm 4.6$ & $84.9 \pm 5.3$ & 0.021 \\
Night diastolic BP, mmHg & $84 \pm 3.4$ & $80.2 \pm 6.5$ & $<0.001$ \\
\hline
\end{tabular}

Table 4 Univariate and multivariate analysis for the parameters affecting cardiac event

\begin{tabular}{|c|c|c|c|c|}
\hline & \multicolumn{2}{|c|}{ Univariate } & \multicolumn{2}{|c|}{ Multivariate } \\
\hline & $\mathbf{p}$ & OR (95\%C.I) & $\mathbf{p}$ & OR (95\%C.I) \\
\hline Age & 0.038 & $\begin{array}{l}1.061(1.033- \\
1.21)\end{array}$ & 0.726 & $\begin{array}{l}0.979(0.869- \\
1.103)\end{array}$ \\
\hline $\begin{array}{l}\text { Diabetes } \\
\text { Mellitus }\end{array}$ & 0.031 & $\begin{array}{l}3.012(1.106- \\
8.198)\end{array}$ & 0.040 & $\begin{array}{l}29.385(1.172- \\
736.770)\end{array}$ \\
\hline $\mathrm{HbAlc} \%$ & 0.006 & $\begin{array}{l}2.146(1.251- \\
3.682)\end{array}$ & 0.595 & $\begin{array}{l}0.683(0.167- \\
2.791)\end{array}$ \\
\hline $\begin{array}{l}\text { LV mass } \\
\text { index }\left(g / \mathrm{m}^{2}\right)\end{array}$ & 0.040 & $\begin{array}{l}1.029(1.001- \\
1.058)\end{array}$ & 0.184 & $\begin{array}{l}1.050(0.977- \\
1.130)\end{array}$ \\
\hline $\begin{array}{l}\text { Sleep-through } \\
\text { surge }\end{array}$ & $<0.001$ & $\begin{array}{l}1.127(1.076- \\
1.181)\end{array}$ & 0.047 & $\begin{array}{l}1.269(1.033- \\
1.606)\end{array}$ \\
\hline
\end{tabular}

OR: Odd's ratio, C.I: Confidence interval, HbAlc: Glycosylated hemoglobin 
Table $5 \&$ Figure 2 show the sensitivity and specificity of the sleepthrough surge in predicting cardiac events in patients with essential hypertension. A sleep-through surge cutoff value $>23 \mathrm{mmHg}$ had sensitivity of $89.5 \%$ and specificity of $81.6 \%$ in predicting cardiac events $(p<0.001)$.

Table 5 Sensitivity, specificity for sleep-trough surge to predict cardiac events

\begin{tabular}{lllllll}
\hline & Cutoff value & Sensitivity & Specificity & PPV & NPV & P \\
\hline Sleep-through surge & $>23 \mathrm{mmHg}$ & 89.47 & 81.58 & 44.7 & 97.9 & $<0.001$ \\
\hline
\end{tabular}

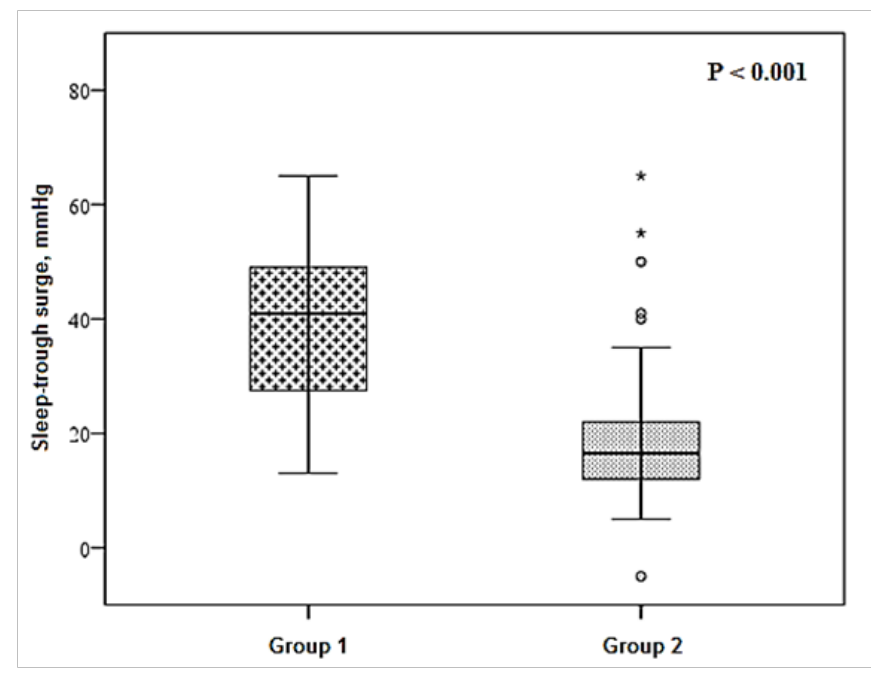

Figure I Sleep-through surge levels in the studied patients.

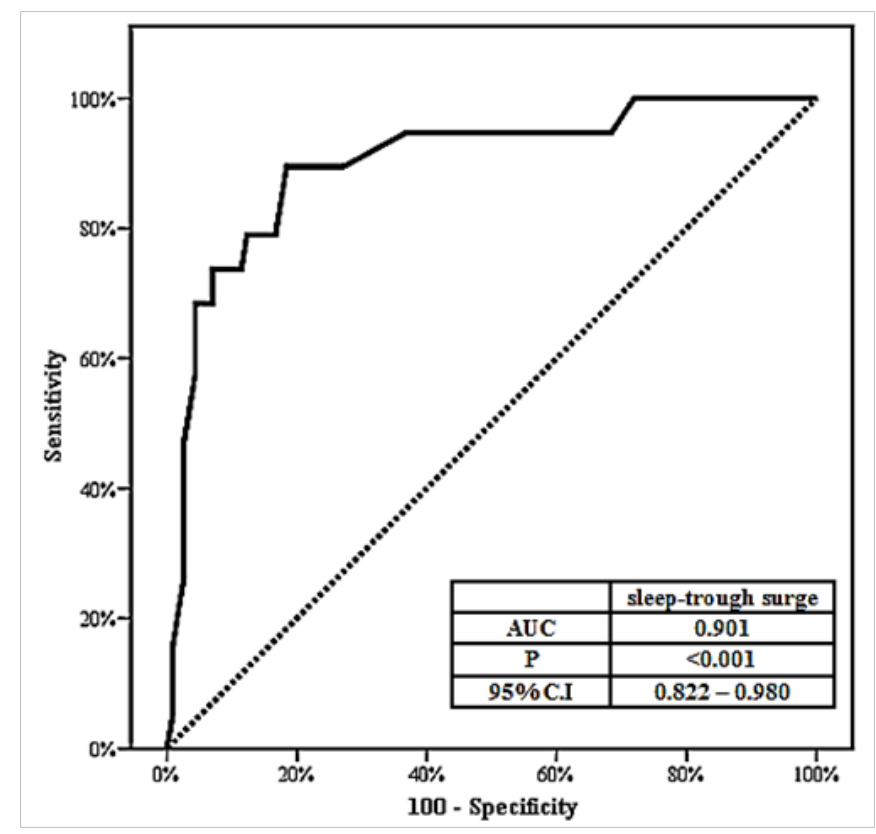

Figure 2 ROC curve for sleep-through surge to predict cardiac events.

AUC:Area under a curve, $\mathrm{p}$ value: Probability value, $\mathrm{Cl}$ : Confidence Intervals

\section{Discussion}

Hypertension is a well-recognized modifiable risk factor for cardiovascular disease through various mechanisms which include LV hypertrophy, endothelial cell injury, impaired LV filling, and, in uncontrolled cases, it could result in systolic heart failure. ${ }^{10}$ Although blood pressure had a diurnal variations (blood pressure is low at night during sleep, but increases a few hours before waking), however, extreme elevations in morning blood pressure are risk factors for cardiovascular disease and are associated with a worse prognosis. ${ }^{11,12}$ Numerous factors are responsible for rises in the morning blood pressure surge. These include increased physical activity, sympathetic overstimulation, renin-angiotensin-aldosterone system activity, elevated cortisol level and disruption in nitric oxide production, causing a further vasoconstrictor effect, with a resultant abrupt rise of blood pressure. ${ }^{13,14}$ The probability of cardiac events, cerebral events and sudden death is increased at the early morning hours after waking from sleep. This could be related to increased morning blood pressure surge. ${ }^{1}$

In the present study, the sleep-through surge was significantly higher in patients who developed cardiac events as compared with those who did not. The data obtained in the present study using the amplitude of the sleep-through surge identified patients at cardiovascular risk. The sensitivity and specificity of a sleep-through surge more than $23 \mathrm{mmHg}$ to predict adverse cardiac events The sensitivity and specificity of a sleep-through surge more than $23 \mathrm{mmHg}$ to predict adverse cardiac events were $89.5 \%$ and $81.6 \%$, respectively.

Gosse et al., ${ }^{15}$ demonstrated the prognostic value of a rise in the blood pressure surge in cardiac events. ${ }^{15}$ Amici et al. reported that a morning systolic blood pressure surge $>34 \mathrm{mmHg}$ was associated with cardiovascular risk. ${ }^{16}$ Similarly, Li et al., ${ }^{17}$ found a significant association between morning blood pressure surge and cardiovascular adverse events. They reported that a morning surge $>37 \mathrm{mmHg}$ was associated with cardiac, cerebral events, and all-cause mortality. ${ }^{17} \mathrm{~A}$ recent large study of 2,020 participants reported that the frequency of increases in sleep-through surges rather than the amplitude of these surges was associated with worse cardiovascular outcomes. ${ }^{18}$

Verdecchia et al., ${ }^{19}$ explored the link between the early morning blood pressure surge and the major cardiovascular events in subjects with an initially untreated essential hypertension. They reported events in $16 \%$ of their subjects during a mean of 8 -y follow-up. However, after adjustment for other significant cofactors (age, sex, smoking, diabetes mellitus, and LV hypertrophy), a blunted, but not elevated morning blood pressure surge was associated with cardiovascular events. They explained the differences between their results and those of previous studies by the white race of the enrolled patients, masked cardiovascular risk factors related to the ethnicity, the patients were not receiving antihypertensive drugs before their participation in the study, and the demographic criteria at entry. ${ }^{19}$

The present study showed that patients who developed cardiac events had an increased LV mass index in comparison with that of patients who did not develop events. However, after a multivariate logistic analysis, this finding was not significant. Cuspid et al., ${ }^{20}$ reported a significant relation between the LV mass index and 
cardiovascular events in a 12-y follow-up study of 1,716 subjects with hypertension.

A morning blood pressure surge is not only a documented risk factor for cardiac events but also for cerebral events. The risk of having a cerebral infarction was proportionate to the amplitude of the morning blood pressure surge. ${ }^{21}$ In another study, a morning blood pressure surge more than $55 \mathrm{mmHg}$ predicted cerebral infarctions in hypertensive patients. ${ }^{22}$ Based on the data in the current study, control of the sleep-through surge in essential hypertension could reduce the incidence of cardiac events.

\section{Conclusion}

Measuring the morning blood pressure surge is feasible in patients with essential hypertension and can provide information about hypertensive patients who are at risk of cardiovascular events.

\section{Funding}

This is no funding authority to declare.

\section{Conflict of interest}

The author declares that they have no conflict of interest.

\section{References}

1. Muller JE, Tofler GH, Stone PH. Circadian variation and triggers of onset of acute cardiovascular disease. Circulation. 1989;79(4):733-743.

2. Sheppard JP, Hodgkinson J, Riley R, et al. Prognostic significance of the morning blood pressure surge in clinical practice: a systematic review. Am J Hypertens. 2015;28(1):30-41.

3. Kario K, White WB. Early morning hypertension: what does it contribute to overall cardiovascular risk assessment?. J Am Soc Hypertens. 2008;2(6):397-402.

4. Gosse P, Cipriano C, Bemurat L, et al. Prognostic significance of BP measured on rising. J Human Hypertens. 2001;15(6):413-417.

5. Neutel JM, Schumacher H, Gosse P, et al. Magnitude of morning blood pressure surge in untreated hypertensive patients: a pooled analysis. Int $J$ Clin Pract. 2008; 62(11):1654-1663.

6. Kario K. Morning surge in blood pressure and cardiovascular risk evidence and perspectives. Hypertension. 2010;56(55):765-773.

7. Iqbal P, Stevenson L. Cardiovascular outcomes in patients with normal and abnormal 24-hour ambulatory blood pressure monitoring. Int $J$ Hypertens.2011;786912.

8. Metoki H, Ohkubo T, Kikuya M, et al. Prognostic significance for stroke of a morning pressor surge and a nocturnal blood pressure decline. Hypertension. 2006;47(2):149-154.
9. Lang RM, Badano LP, Mor-Avi V, et al. Recommendations for Cardiac Chamber Quantification by Echocardiography in Adults: An Update from the American Society of Echocardiography and the European Association of Cardiovascular Imaging. J Am Soc Echocardiogr. 2015;28(1):1-39.

10. Lawes CM, Vander Hoorn S, Rodgers A, et al. Global burden of bloodpressure-related disease, 2001. Lancet. 2008;371(9623):1513-1518.

11. Amodeo C, Guimarães GG, Picotti JC, et al. Morning blood pressure surge is associated with death in hypertensive patients. Blood Press Monit. 2014;19(4):199-202.

12. Kario K. Morning surge in blood pressure and cardiovascular risk: evidence and perspectives. Hypertension. 2010;56(55):765-773.

13. Kario K, Yano Y, Matsuo T, et al. Additional impact of morning haemostatic risk factors and morning blood pressure surge on stroke risk in older Japanese hypertensive patients. Eur Heart J. 2011;32(5):574580 .

14. Metoki H, Ohkubo T, Kikuya M, et al. Prognostic significance for stroke of a morning pressor surge and a nocturnal blood pressure decline. Hypertension. 2006; 47(2):149-154.

15. Gosse P, Lasserre R, Minifie C, et al. Blood pressure surge on rising. $J$ Hypertens. 2004; 22:1113-1118.

16. Amici A, Cicconetti P, Sagrafoli C, et al. Exaggerated morning blood pressure surge and cardiovascular events: a 5-year longitudinal study in normotensive and well-controlled hypertensive elderly. Arch Gerontol Geriatr. 2009;49:105-109.

17. Li Y, Thijs L, Hansen TW, et al. International Database on Ambulatory Blood Pressure Monitoring in Relation to Cardiovascular Outcomes Investigators. Prognostic value of the morning blood pressure surge in 5645 subjects from 8 populations. Hypertension. 2010; 55(4):1040-1048.

18. Cheng HM, Wu CL, Sung SH, et al. Prognostic Utility of Morning Blood Pressure Surge for 20-Year All-Cause and Cardiovascular Mortalities: Results of a Community-Based Study. J Am Heart Assoc. 2017;6 (12).

19. Verdecchia P, Angeli F, Mazzotta G, et al. Day-night dip and earlymorning surge in blood pressure in hypertension: prognostic implications. Hypertension. 2012;60(1):34-42.

20. Cuspidi C, Facchetti R, Bombelli M, et al. Differential value of left ventricular mass index and wall thickness in predicting cardiovascular prognosis: data from the PAMELA population. Am J Hypertens. 2014; 27(8):1079-1086.

21. Kario K, Shimada K, Pickering TG.Clinical implications of morning blood pressure surge in hypertension. J Cardiovasc Pharmacol. 2003; 42(Supply 1): S87-91.

22. Kario K, Pickering TG, Umeda Y, et al. Morning surge in blood pressure as a predictor of silent and clinical cerebrovascular disease in elderly hypertensives. Circulation. 2003;107(10):1401-1406. 